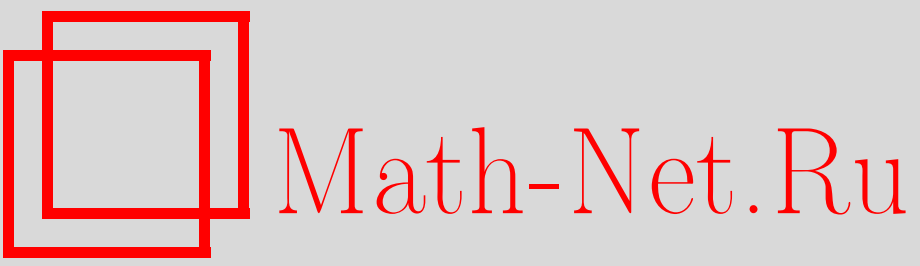

Г. А. Мартынов, Гидродинамическая теория распространения звуковых волн, TMФ, 2001, том 129, номер 1, 140-152

DOI: https://doi.org/10.4213/tmf526

Использование Общероссийского математического портала Math-Net.Ru подразумевает, что вы прочитали и согласны с пользовательским соглашением

http://www . mathnet.ru/rus/agreement

Параметры загрузки:

IP : 18.209 .158 .208

26 апреля 2023 г., 14:45:54 


\author{
ТЕОРЕТИЧЕСКАЯ \\ И МАТЕМАТИЧЕСКАЯ \\ ФИЗИКА \\ Том 129, № 1 \\ октябрь, 2001
}

(C) 2001 г.

Г.А. Мартынов*

\title{
ГИДРОДИНАМИЧЕСКАЯ ТЕОРИЯ РАСПРОСТРАНЕНИЯ ЗВУКОВЫХ ВОЛН
}

\begin{abstract}
Сформулированы границы применимости уравнений гидродинамики и обоснована необходимость введения в уравнение теплопроводности поправки на перенос потенциальной энергии. С учетом этой поправки разработана гидродинамическая теория распространения звуковых волн малой амплитуды и показано, что она существенно меняет почти все предсказания классической гидродинамической теории. В частности, введенная поправка позволяет распространить область применимости гидродинамической теории на случай сколь угодно вязких жидкостей. Кроме того, в полном согласии с экспериментом теория предсказывает, что скорость звука и коэффициент затухания остаются конечными при всех частотах вплоть до частот порядка $10^{-12} \mathrm{c}^{-1}$, при больших частотах уравнения гидродинамики теряют смысл и, как следствие, распространение звуковой волны в среде становится невозможным. Показано, что безразмерное дисперсионное уравнение содержит всего один материальный параметр. Предсказана возможность существования сильно затухающего второго звука.
\end{abstract}

\section{1. ВВЕДЕНИЕ}

Как известно, уравнения гидродинамики представляют собой математическую формулировку законов сохранения массы, импульса и энергии для сплошной среды. Поэтому они должны описывать макроскопические процессы совершенно точно, но, конечно, до тех пор, пока применима сама модель сплошной среды. Однако если мы начнем решать уравнения гидродинамики для простейшей задачи распространения звуковой волны малой амплитуды путем разложения по волновому вектору $k$, то обнаружим, что уже во втором приближении по этому параметру они приводят к формуле Кирхгофа для коэффишиента затухания $x$, согласно которой $x$ растет линейно с ростом вязкости $\eta$ [1]. Этот результат противоречит опытным данным, так как из него следует, что в вязких жидкостях звук практически вообще не должен распространяться. Более того, численный анализ точного дисперсионного уравнения, полученного из уравнений гидродинамики, проведенный Трусделлом, показал, что гидродинамическая теория звука ни при каких значениях параметров не может описывать экспериментальные данные [2], [3].

Дальнейшее развитие теории пошло по пути отказа от модели сплошной среды. С одной стороны, были предприняты попытки получения дисперсионного уравнения на основе уравнения Больцмана (более радикальньй вариант, при котором рассмотрение

\footnotetext{
${ }^{*}$ Институт физической химии РАН, Москва, Россия. E-mail:martynov@lmm.phyche.msk.su
} 
целиком переносится на микроуровень, см. в книге [4]), с другой - предлагалось внести элементы микротеории в макроскопические уравнения гидродинамики (менее радикальный вариант). В последнем случае исследования привели к созданию так называемой релаксационной теории [3], согласно которой энергия звуковых волн может передаваться от внешних (макроскопических) степеней свободы к внутренним (микроскопическим) степеням свободы. При этом возможны два механизма: поглощение звуковой энергии отдельными атомами, колеблющимися внутри многоатомных молекул, и передача энергии от одних более протяженных структур жидкости к другим, менее протяженным. Первьй механизм заведомо не может быть обшим, поскольку расхождение теории с экспериментом наблюдается как в сложных молекулярных, так и в простых одноатомных жидкостях, в которых внутримолекулярные степени свободы вообще отсутствуют. Второй механизм требует более детального анализа.

В то время когда создавалась релаксационная теория (т.е. почти 50 лет тому назад), представления о структуре жидкости были весьма расплывчатыми. Теперь ситуация изменилась: можно уверенно утверждать, что в жидкости существует только одна структура, описываемая двухчастичной функцией распределения [4], [5]. Эта функция обрашается в единицу на расстояниях $R \approx 10^{-7}$ см от выделенной частицы, вырезая тем самым вокруг нее корреляционную сферу. Согласно современным представлениям статистической механики свойства жидкости однозначно определяются распределением плотности вещества внутри корреляционной сферы; все, что происходит вне ее, на параметры жидкости не влияет [5]. Наличие всего одной структуры уже само по себе исключает возможность обмена энергией между разными структурами. Но, что не менее важно, из-за малости размеров корреляционной сферы становится невозможным поглошение энергии звуковых колебаний самой этой сферой. Действительно, в ультразвуковом диапазоне, где расхождение между теорией и экспериментом весьма значительно, длина звуковой волны $l \approx 10^{-1}$ см на шесть (!) порядков превосходит размеры корреляционной сферы $R \approx 10^{-7}$ см. При таком различии длин резонансные эффекты вряд ли возможны ${ }^{1)}$. Кроме того, поскольку средняя тепловая скорость движения молекул $v=\sqrt{2 \pi \theta / m} \approx 10^{5} \mathrm{~cm} / \mathrm{c}$ (здесь $\theta=k_{\mathrm{B}} T$ - температура в энергетических единицах), то время установления термодинамического равновесия внутри корреляционной сферы $\tau \approx R / v \approx 10^{-12}$ с. Поэтому, если характерное время макроскопического процесса $t_{0} \gg \tau$, вешество внутри корреляционной сферы успевает прийти в состояние термодинамического равновесия с окружающей средой и обмен энергией между вешеством и средой прекращается. На этом фактически и основано приближение локального равновесия, с помощњю которого уравнения гидродинамики выводятся из иерархии ББГКИ [5].

Итак, модель сплошной среды должна быть справедлива при выполнении двух неравенств

$$
l \gg 10^{-7} \mathrm{~cm}, \quad t \gg 10^{-12} \mathrm{c} .
$$

Каждое из них с громадным запасом выполняется в звуковом и ультразвуковом диа-

\footnotetext{
1) Приведу пример. Предположим, что бутылка с водой плавает на поверхности океана, по поверхности которого бегут волны, длина которых $l$ на шесть порядков превосходит размеры бутылки. Очевидно, что в этом случае уровень воды в бутылке останется неподвижным (по отношению к ее стенкам) и передача энергии от вод океана к воде в бутылке окажется невозможной.
} 
пазонах; они могут быть нарушены только в области гиперзвуковых частот, где $\omega \approx$ $10^{11} \mathrm{c}^{-1}$.

Проведенные вьше оценки показывают, что привлечение элементов микротеории для объяснения процесса распространения звуковых волн является излишним, из-за того что длина звуковой волны, как правило, намного превосходит любой микроскопический размер (в том числе и длину свободного пробега). Но тогда чем же объясняется расхождение гидродинамической теории с экспериментом, столь отчетливое уже в ультразвуковом диапазоне?

В данной статье будет показано, что одно из трех уравнений гидродинамики, уравнение теплопроводности, обычно записывается не совсем точно: в нем учитывается поток кинетической энергии частиц $\vec{J}=-\lambda \partial \theta / \partial \vec{r}$ (закон Фурье $)^{2)}$ и не учитывается поток потенциальной энергии. Если в уравнение теплопроводности ввести соответствующий член, обобшенная таким образом гидродинамическая теория приходит, как мы увидим, в согласие с экспериментом.

\section{2. УРАВНЕНИЯ ТЕПЛОПРОВОДНОСТИ}

Из неравновесной иерархии ББГКИ (которая, в свою очередь, является следствием уравнений Ньютона, описывающих движение каждой частищы вешества в отдельности) может быть получено точное выражение для закона сохранения внутренней энергии [4],

$$
\rho \frac{d e}{d t}=-\frac{\partial J_{\alpha}}{\partial r_{\alpha}}-P_{\alpha \beta} D_{\alpha \beta}
$$

Здесь по определению $e=3 \theta / 2+\bar{\Phi}-$ внутренняя энергия одной частишы, $\theta=$ $\overline{(\vec{p}-m \vec{v})^{2} / 2 m}$ - температура, равная средней кинетической энергии хаотического движения частицы, $\vec{p}$ - импульс частицы, $\vec{v}$ - скорость упорядоченного движения жидкости, $\bar{\Phi}$ - потенциальная энергия взаимодействия частицы со своим окружением, $\rho$ - плотность жидкости, $t-$ время, $\vec{r}$ - координата, $P_{\alpha \beta}-$ тензор натяжений, $\vec{J}$ - поток внутренней энергии и

$$
\frac{d}{d t}=\frac{\partial}{\partial t}+v_{\alpha} \frac{\partial}{\partial r_{\alpha}}, \quad D_{\alpha \beta}=\frac{1}{2}\left(\frac{\partial v_{\alpha}}{\partial r_{\beta}}+\frac{\partial v_{\beta}}{\partial r_{\alpha}}\right) .
$$

При выполнении неравенств (1) в системе реализуется состояние локального термодинамического равновесия, при котором $e=e(\rho(r, t), \theta(r, t))$, где $\rho=\rho(\vec{r}, t)$ - локальная плотность вешества, $\theta(\vec{r}, t)$ - локальная температура. В этом приближении

$$
\rho \frac{d e}{d t} \equiv \rho c_{V} \frac{d \theta}{d t}+\rho\left(\frac{\partial e}{\partial \rho}\right)_{\theta} \frac{d \rho}{d t}=-\frac{\partial J_{\alpha}}{\partial r_{\alpha}}-P_{\alpha \beta} D_{\alpha \beta},
$$

где теплоемкость при постоянном объеме $c_{V}=(\partial e / \partial \theta)_{\rho}$.

Выделим теперь из тензора давлений $P_{\alpha \beta}$ и из тензора скоростей $D_{\alpha \beta}$ недиагональные компоненты $\widetilde{P}_{\alpha \beta}, \widetilde{D}_{\alpha \beta}$, положив

$$
P_{\alpha \beta}=\widetilde{P}_{\alpha \beta}+\delta_{\alpha \beta} P, \quad D_{\alpha \beta}=\widetilde{D}_{\alpha \beta}+\delta_{\alpha \beta} \frac{\partial v_{\gamma}}{\partial r_{\gamma}},
$$

\footnotetext{
2) Напомню, что согласно представлениям статистической механики температура $\theta$ имеет смысл средней кинетической энергии, приходящейся на одну степень свободы частицы. Поэтому закон Фурье описывает процесс переноса кинетической энергии.
} 
где $\delta_{\alpha \beta}-$ символ Кронекера. В этом случае

$$
P_{\alpha \beta} D_{\alpha \beta}=\widetilde{P}_{\alpha \beta} \widetilde{D}_{\alpha \beta}+P \frac{\partial v_{\alpha}}{\partial r_{\alpha}},
$$

где $P=P(\rho, \theta)$ - локальное давление. Так как из уравнения непрерывности следует, что

$$
\frac{\partial v_{\alpha}}{\partial r_{\alpha}}=-\frac{1}{\rho} \frac{d \rho}{d t}
$$

то уравнение баланса внутренней энергии (2) в приближении локального равновесия принимает вид

$$
\rho c_{V} \frac{d \theta}{d t}+\rho\left(\frac{\partial e}{\partial \rho}\right)_{\theta} \frac{d \rho}{d t}=-\frac{\partial J_{\alpha}}{d r_{\alpha}}+\frac{P}{\rho} \frac{d \rho}{d t}-\widetilde{P}_{\alpha \beta} \widetilde{D}_{\alpha \beta}
$$

Предположим сначала, что поток внутренней энергии определяется законом Фурье $\vec{J}=$ $-\lambda(d \theta / d \vec{r})$, и рассмотрим произвольньй одномерный изотермический процесс, при котором $d \theta / d t=0, J=0$ и $\widetilde{P}_{\alpha \beta}=\widetilde{D}_{\alpha \beta}=0$. В этом случае уравнение (7) после сокрашения на $d \rho / d t$ сводится к

$$
\rho^{2}\left(\frac{\partial e}{\partial \rho}\right)_{\theta}=P
$$

что, однако, противоречит известному термодинамическому тождеству (см. формулу (16.5) в $[6])$

$$
\rho^{2}\left(\frac{\partial e}{\partial \rho}\right)_{\theta}=P-\theta\left(\frac{\partial P}{\partial \theta}\right)_{\rho} .
$$

Итак, уравнение теплопроводности, полученное в приближении Фурье, в данном случае приводит к результатам, противоречащим термодинамике. В релаксационной теории равновесная теплоемкость $c_{V}=c_{V}(\rho, \theta)$ в (7) заменяется на теплоемкость, зависящую от частоты звуковой волны $\omega$. Однако это не устраняет возникшего противоречия, поскольку в изотермических процессах теплоемкость вообще выпадает из уравнения теплопроводности.

Заметим теперь, что все входяшие в уравнение (7) функции, в том числе и поток тепла $\vec{J}$, зависят от координаты $\vec{r}$. Поэтому всегда можно написать, что $\vec{J}=-\partial Q / \partial \vec{r}$. Для того чтобы это выражение совпало с законом Фурье, необходимо предположить, что производящая функция имеет вид $Q=Q(\theta(\vec{r}))$. В этом случае

$$
\vec{J}=-\frac{\partial Q}{\partial \theta} \frac{\partial \theta}{\partial \vec{r}}
$$

откуда коэффициент теплопроводности $\lambda=\partial Q / \partial \theta$. Но эксперимент однозначно указывает на то, что теплопроводность зависит не только от температуры, но и от плотности вешества, т.е. $\lambda=\lambda(\rho, \theta)$. Подобная зависимость возможна лишь в том случае, когда $Q$ является функцией не только температуры, но и плотности, т.е. $Q=Q(\rho, \theta)$. Впрочем, подобную зависимость можно было предположить и не апеллируя к эксперименту, поскольку в состоянии термодинамического равновесия, в том числе и локального, все параметры вешества (давление $P$, теплоемкость $c_{V}$ и т.д.) являются функциями $\rho$ и $\theta$. 
Из формулы $Q=Q(\rho, \theta)$ автоматически следует, что поток внутренней энергии (именно энергии, а не тепла!) ${ }^{3)}$

$$
\vec{J}=-\lambda \frac{\partial \theta}{\partial \vec{r}}+\chi \frac{\partial \rho}{\partial \vec{r}}
$$

где $\chi=\partial Q / \partial \rho$. Впервые это выражение в общем виде было получено в работе [5] на основе иерархии ББГКИ, т.е. исходя из фундаментальных представлений статистической механики. Для газов оно было получено ранее на основе уравнения Больцмана [7], [8].

Чтобы лучше понять физический смысл второго члена в (10), рассмотрим стационарное течение газа в трубе, соединяюшей два резервуара А и В, температура которых одинакова. Будем считать, что в каждом из резервуаров газ находится в состоянии термодинамического равновесия. Для того чтобы газ перетекал из резервуара А в резервуар $\mathrm{B}$, необходимо, чтобы давление в первом резервуаре было больше, чем во втором. Но в состоянии равновесия $P=P(\rho)$. Поэтому перетекание газа из одного резервуара в другой возможно только при условии, что $\rho_{\mathrm{A}}>\rho_{\mathrm{B}}$. А так как внутренняя энергия зависит от плотности вешества, $e=e(\rho)$, то при перемешении одной частицы из А в $\mathrm{B}$ ее энергия изменится на величину

$$
e\left(\rho_{\mathrm{A}}\right)-e\left(\rho_{\mathrm{B}}\right) \approx \frac{\partial e}{\partial \rho}\left(\rho_{\mathrm{A}}-\rho_{\mathrm{B}}\right) .
$$

Благодаря этому между резервуарами возникает дополнительный поток внутренней энергии (не тепла, а именно внутренней энергии!), пропорциональный градиенту плотности. Подобная зависимость обусловлена тем, что величина $\rho$ определяет среднее расстояние $\bar{r}$ между частицами и тем самым задает среднее значение потенциальной энергии взаимодействия частиц $\bar{\Phi} \approx \Phi(\bar{r})$. Учитьвая вышесказанное, будем называть коэффициент пропорциональности $\chi$ коэффициентом потенциалопроводности. В выражении (10) перед $\chi$ поставлен знак плюс потому, что плотность потенциальной энергии растет с увеличением плотности вешества. Соответственно, ее поток направлен в ту же сторону, что и градиент плотности (т.е. от областей с меньшей плотностью в сторону областей с большей плотностью), тогда как в случае кинетической энергии поток направлен противоположно градиенту температуры (т.е. от более высокой температуры к более низкой).

Подставляя (10) в (7) и учитывая термодинамическое тождество (9), представим уравнение баланса внутренней энергии (уравнение теплопроводности) в виде

$$
\rho c_{V} \frac{d \theta}{d t}-\frac{\theta P_{\theta}}{\rho} \frac{d \rho}{d t}=\lambda \Delta \theta-\chi \Delta \rho-\widetilde{P}_{\alpha \beta} \widetilde{D}_{\alpha \beta}
$$

Деля обе части уравнения (11) на $\rho c_{V}$, получим

$$
\frac{d \theta}{d t}-g \frac{d \rho}{d t}=\alpha \Delta \theta-b \Delta \rho-\frac{1}{\rho c_{V}} \widetilde{P}_{\alpha \beta} \widetilde{D}_{\alpha \beta}
$$

\footnotetext{
3) Уравнение (2) обычно называют уравнением баланса тепла или просто уравнением теплопроводности. Но что такое тепло и почему оно должно сохраняться? На этот вопрос статистическая механика не дает ответа. В отличие от этого понятие "энергия" в статистической механике определено совершенно точно, и нет сомнений, что энергия должна сохраняться.
} 
где $\alpha=\lambda / \rho c_{V}-$ коэффициент температуропроводности, $b=\chi / \rho c_{V}$ и $g=\theta P_{\theta} / \rho^{2} c_{V}-$ константы, $P_{\theta}=(\partial P / \partial \theta)_{\rho}$.

Выражения, стоящие в левой части (11), учитывают как кинетическую, так и потенциальную энергию, которая фиксируется внутри физически бесконечно малого объема $\delta V$. Соответственно в правой части (11) член с $\Delta \theta$ описывает приток кинетической энергии в $\delta V$ за счет ее поступления из окружаюшей среды, член с $\Delta \rho$-приток извне потенциальной энергии, а последний член $\widetilde{P}_{\alpha \beta} \widetilde{D}_{\alpha \beta}$ учитьвает изменение внутренней энергии в результате процессов диссипации, происходяших в самом объеме $\delta V$. Введение дополнительного члена в уравнение баланса энергии (11) можно объяснить следуюшим образом. Если мы учли зависимость внутренней энергии $e$ от $\rho$ в левой части уравнения, то в правой части того же уравнения мы должны учесть поток массы через поверхность, ограничивающую $\delta V$, поскольку именно этот поток вызывает изменение плотности вещества внутри $\delta V$.

\section{3. ДИСПЕРСИОННОЕ УРАВНЕНИЕ}

Уравнение непрерывности (6), уравнение Навье-Стокса

$$
\rho \frac{d v_{\alpha}}{d t}=P_{\rho} \frac{\partial \rho}{\partial r_{\alpha}}+P_{\theta} \frac{\partial \theta}{\partial r_{\alpha}}+\tilde{\eta} \Delta v_{\alpha}+\left(\tilde{\xi}+\frac{1}{3} \tilde{\eta}\right) \frac{\partial}{\partial r_{\alpha}} \frac{\partial v_{\beta}}{\partial r_{\beta}}
$$

и уравнение баланса внутренней энергии (12) образуют замкнутую систему трех уравнений для определения трех гидродинамических переменных $\rho(r, t), v(r, t)$ и $\theta(r, t)$ (здесь $\tilde{\xi}$ - объемная, а $\tilde{\eta}$ - сдвиговая вязкость, $\left.P_{\rho}=(\partial P / \partial \rho)_{\theta}\right)$. В дальнейшем при решении этой системы мы ограничимся рассмотрением звука малой амплитуды, при котором отклонения $\delta \rho, \delta v$ и $\delta \theta$ от равновесных значений $\rho_{0}, v_{0}, \theta_{0}=$ const достаточно малы,

$$
\rho(r, t)=\rho_{0}+\delta \rho(r, t), \quad v(r, t)=v_{0}+\delta v(r, t), \quad \theta(r, t)=\theta_{0}+\delta \theta(r, t) .
$$

В этом случае в $(8),(12)$ и (13) можно пренебречь квадратичными членами по $\delta \rho, \delta v$ и $\delta \theta$, после чего с помощью уравнения непрерывности можно исключить из уравнений скорость движения жидкости $\delta v$. В результате система трех уравнений сводится к системе двух уравнений для $\delta \rho$ и $\delta \theta$

$$
\begin{gathered}
{\left[\frac{\partial^{2}}{\partial t^{2}}-\left(\eta \frac{\partial}{\partial t}+P_{\rho}\right) \Delta\right] \delta \rho(r, t)=P_{\theta} \Delta \delta \theta(r, t),} \\
{\left[g \frac{\partial}{\partial t}-b \Delta\right] \delta \rho(r, t)=\left[\frac{\partial}{\partial t}-a \Delta\right] \delta \theta(r, t),}
\end{gathered}
$$

где $\Delta$ - лапласиан, $a=\lambda /\left(\rho c_{V}\right)$ - коэффишиент температуропроводности и константы

$$
\begin{gathered}
\eta=\frac{1}{\rho}\left(\tilde{\zeta}+\frac{4}{3} \tilde{\eta}\right)>0, \quad g=\frac{\theta P_{\theta}}{\rho^{2} c_{V}}>0, \quad b=\frac{\chi}{\rho c_{V}}>0, \\
P_{\rho}=\left(\frac{\partial P}{\partial \rho}\right)_{\theta}>0, \quad P_{\theta}=\left(\frac{\partial P}{\partial \theta}\right)_{\rho}>0 .
\end{gathered}
$$

Уравнения (15), (16) отличаются от тех, которые рассматривались Трусделлом [2], только дополнительным членом $b \Delta$, введенным выше. 
Решение системы уравнений (15), (16) будем искать в виде волн плотности и температуры, бегуших вдоль оси $z$,

$$
\delta \rho=A e^{i(\omega t-\tilde{k} z)}, \quad \delta \theta=B e^{i(\omega t-\tilde{k} z)}
$$

где $\tilde{k}=k-i x, k-$ волновой вектор и $x-$ коэффициент затухания. В этих обозначениях скорость звука определяется как $c=\omega / k$.

Подставляя (18) в (16), получим соотношение между амплитудами

$$
B=\frac{b \tilde{k}^{2}+i \omega g}{a \tilde{k}^{2}+i \omega} A
$$

Исключая затем с помощью этого выражения амплитуду $B$ из $\delta \theta$ и подставляя (18) в (15), получим после сокрашения на $A$ комплексное дисперсионное уравнение

$$
-\omega^{2}+\tilde{k}^{2}\left(i \eta \omega+P_{\rho}\right)=-\tilde{k}^{2} P_{\theta} \frac{b \tilde{k}^{2}+i g \omega}{a \tilde{k}^{2}+i \omega}
$$

Приравнивая нулю действительную и мнимую части (20), преобразуем его в систему двух действительных алгебраических уравнений

$$
\begin{gathered}
\tilde{p} c^{2} k^{2}\left(k^{2}-x^{2}\right)-2 c k^{2} x\left(2 a \eta\left(k^{2}-x^{2}\right)+P_{S}\right)-\tilde{q}\left(k^{4}-6 k^{2} x^{2}+x^{4}\right)=0, \\
c^{3} k^{2}-2 \tilde{p} c^{2} k^{2} x-c\left(a \eta\left(k^{4}-6 k^{2} x^{2}+x^{4}\right)+P_{S}\left(k^{2}-x^{2}\right)\right)+4 \tilde{q} k\left(k^{2}-x^{2}\right)=0,
\end{gathered}
$$

где

$$
\tilde{p}=a+\eta, \quad \tilde{q}=a P_{\rho}+b P_{\theta}, \quad P_{S}=\left(\frac{\partial P}{\partial \rho}\right)_{S}=\gamma P_{\rho}, \quad \gamma=\frac{c_{P}}{c_{V}}
$$

и $S$ - энтропия, $c_{P}$ - теплоемкость при постоянном давлении. В первоначальные выражения ни $P_{S}$, ни $c_{P}$ не входили - они появились в результате применения известной формулы термодинамики [6], согласно которой

$$
\theta \frac{P_{\theta}^{2}}{P_{\rho}}=c_{P}-c_{V}=c_{V}(\gamma-1)
$$

Подчеркнем, что дисперсионные уравнения $(21),(22)$ являются точным решением уравнений (15), (16). Последние, в свою очередь, были получены путем тождественных преобразований из уравнений гидродинамики в приближении малости амплитуды звуковой волны и, следовательно, являются асимптотически точными выражениями законов сохранения массы, импульса и внутренней энергии. 


\section{4. ЗВУКОВЫЕ И УЛЬТРАЗВУКОВЫЕ ЧАСТОТЫ}

При не очень высоких частотах $\omega \leqslant 10^{7} \mathrm{c}^{-1}$ (звуковой и ультразвуковой диапазоны) волновой вектор $k$ можно считать малым параметром. В этом случае решение дисперсионных уравнений удобно искать в виде рядов по степеням $k^{4}$

$$
c=c_{0}+c_{2} k^{2}+c_{4} k^{4}+\cdots, \quad x=x_{0}+x_{2} k^{2}+x_{4} k^{4}+\cdots .
$$

Подставляя эти выражения в уравнения $(20),(21)$ и приравнивая члены одного порядка малости по $k$, получим

$$
\begin{gathered}
c_{0}=\sqrt{P_{S}}=\sqrt{\gamma P_{\rho}}, \quad x_{0}=0, \\
A \equiv 2 c_{0} x_{2}=a\left(1-\frac{1}{\gamma}\right)+\eta-\frac{b P_{\theta}}{\gamma P_{\rho}}, \quad B \equiv 2 c_{0} c_{2}=a \eta-(a+\eta) A+\frac{7}{4} A^{2}, \\
x_{4}=-\frac{1}{\left(2 c_{0}\right)^{3}}\left(8 a \eta A+A\left(6 A^{2}+2 B\right)-(a+\eta)\left(5 A^{2}+4 B\right)\right) .
\end{gathered}
$$

Выражение для $c_{4}$ мы не приводим, так как обычно в ультразвуковом и звуковом диапазонах изменения скорости звука достаточно малы [3].

Формула (26) для нулевой скорости звука в точности совпадает с формулой Лапласа. Но уже выражение (27) для коэффициента затухания $A$ отличается от известной формулы Кирхгофа [1]

$$
A=a\left(1-\frac{1}{\gamma}\right)+\eta
$$

слагаемым $b P_{\theta} /\left(\gamma P_{\rho}\right)$, что качественно меняет результат. Действительно, согласно формуле Кирхгофа коэффициент затухания звука растет линейно с ростом вязкости $\eta=(\tilde{\xi}+4 \tilde{\eta} / 3)$. Поэтому согласно $(29)$ в вязких жидкостях звук вообще не должен был бы распространяться, что, однако, не соответствует действительности (напомню, что вязкость $\tilde{\eta}$ может меняться чуть ли не на 15 порядков; значения $\tilde{\xi}$ нам сейчас фактически неизвестны). В то же время появление в (27) отрицательного члена делает возможным компенсацию вязкостного члена и тем самым приводит теорию в соответствие с экспериментом.

Разрешая (27) относительно $\eta$ и $b$, получим

$$
\eta=A+\frac{3 A^{2}-4 B}{4(A-a)}, \quad b \frac{P_{\theta}}{\gamma P_{\rho}}=a\left(1-\frac{1}{\gamma}\right)+\eta-A .
$$

Отсюда следует, что с ростом вязкости коэффициент затухания $A \rightarrow a$, а это означает, что в очень вязких жидкостях поглошение звуковой энергии в основном определяется тепловыми процессами. Из (30) также следует, что, измерив значения $A$ и $B$, можно затем по этим формулам рассчитать объемную вязкость $\tilde{\xi}$ и коэффициент потенциалопроводности $\chi$.

\footnotetext{
${ }^{4)}$ На самом деле разложение ведется по безразмерному вектору $K=k \sqrt{a \eta} / c_{0}$ (см. ниже).
} 
Так как обычно в звуковом и ультразвуковом диапазонах скорость звука меняется мало, то в хорошем приближении можно положить $B=0$. В этом случае получаем совсем простые выражения

$$
\eta=A+\frac{3 A^{2}}{4(A-a)}, \quad b \frac{P_{\theta}}{\gamma P_{\rho}}=a\left(1-\frac{1}{\gamma}\right)+\frac{3 A^{2}}{4(A-a)}
$$

для определения неизвестных констант $\tilde{\xi}$ и $\chi$.

\section{5. АНАЛИЗ ДИСПЕРСИОННЫХ УРАВНЕНИЙ}

Введем безразмерные переменные

$$
C=\frac{c}{c_{0}}, \quad X=\frac{x}{k}, \quad K=\frac{k}{k_{0}}, \quad \text { где } \quad k_{0}=\frac{c_{0}}{\sqrt{a \eta}} .
$$

В этих переменных дисперсионные уравнения запишутся в виде системы двух квадратных уравнений относительно безразмерного волнового вектора $K$,

$$
\begin{gathered}
4 C X\left(1-X^{2}\right) K^{2}-\left(p\left(1-X^{2}\right) C^{2}-q\left(1-6 X^{2}+X^{4}\right)\right) K+2 C X=0 \\
C\left(1-6 X^{2}+X^{4}\right) K^{2}-4 q\left(1-X^{2}\right) K-C\left(C^{2}-2 p X C-\left(1-X^{2}\right)\right)=0
\end{gathered}
$$

где

$$
p=\frac{a+\eta}{\sqrt{a \eta}}, \quad q=\frac{a P_{\rho}+b P_{\theta}}{P_{S} \sqrt{a \eta}}=\frac{1}{\sqrt{a \eta}}\left(\frac{a}{\gamma}+b \frac{P_{\theta}}{\gamma P_{\rho}}\right) .
$$

Подставляя в $(35)$ вместо $b\left(P_{\theta} / P_{\rho}\right)$ его выражение из $(30)$, получим

$$
q=\frac{a+\eta-A}{\sqrt{a \eta}} .
$$

Чтобы выяснить, как в этом выражении меняется величина $A$, обратимся к первому равенству (30), из которого следует, что при $\eta=0$ константа $A=A_{\min }$ является решением квадратного уравнения $7 A^{2}-4 a A-4 B=0$. Отсюда получим, что

$$
A_{\min }=\frac{2 a}{7}\left(1+\sqrt{1+7 \frac{B}{a^{2}}}\right)=\frac{4 a}{7}+\frac{B}{a^{2}}+\cdots .
$$

$\mathrm{C}$ другой стороны, при $\eta=\infty$ величина $A=A_{\max }$, где

$$
A_{\text {max }}=a \text {. }
$$

Таким образом, значения приведенного коэффициента затухания $A$ лежат в интервале

$$
a \geqslant A>\frac{4}{7} a+\frac{B}{a^{2}},
$$

т.е. меняются в очень ограниченных пределах, оставаясь почти равными коэффициенту температуропроводности $a$. Поэтому без большой ошибки можно положить $A=a$, что дает

$$
p=\sqrt{\frac{\eta}{a}}+\sqrt{\frac{a}{\eta}} \approx q+\frac{1}{q}, \quad q \approx \sqrt{\frac{\eta}{a}} .
$$


Таким образом, безразмерные дисперсионные уравнения (33) и (34) фактически содержат лишь один материальный параметр $q$.

Анализируя полученные выше выражения, можно сформулировать еше несколько полезных неравенств. С этой целью решим каждое из квадратных уравнений $(33),(34)$ относительно $K$,

$$
\begin{aligned}
K^{(1)}= & \frac{1}{8 C X\left(1-X^{2}\right)}\left\{\left(p\left(1-X^{2}\right) C^{2}-q\left(1-6 X^{2}+X^{4}\right)\right)-\right. \\
& \left.-\sqrt{\left(p\left(1-X^{2}\right) C^{2}-q\left(1-6 X^{2}+X^{4}\right)\right)^{2}-32 C^{2} X^{2}\left(1-X^{2}\right)}\right\}, \\
K^{(2)}= & \frac{1}{C\left(1-6 X^{2}+X^{4}\right)}\left\{2 q\left(1-X^{2}\right)-\right. \\
& \left.-\sqrt{4 q^{2}\left(1-X^{2}\right)^{2}+C^{2}\left(1-6 X^{2}+X^{4}\right)\left(C^{2}-2 p X C-\left(1-X^{2}\right)\right)}\right\},
\end{aligned}
$$

знаки в решениях $(41),(42)$ выбраны таким образом, чтобы в пределе нулевых частот выполнялись условия $C=1, X=K=0$ (см. (26)). Очевидно, что система уравнений (41), (42) имеет решение только при $K^{(1)}=K^{(2)}$.

Для того чтобы волновой вектор $K^{(2)}$ был положительным, необходимо, чтобы произведение $\left(1-6 X^{2}+X^{4}\right)\left(C^{2}-2 p X C-\left(1-X^{2}\right)\right)$, стоящее под знаком корня в $(42)$, было отрицательным. Это возможно в двух случаях:

$$
\begin{aligned}
& \text { a) } \quad 1-6 X^{2}+X^{4}>0, \quad C^{2}-2 p X C-\left(1-X^{2}\right)<0 \text {, } \\
& \text { б) } 1-6 X^{2}+X^{4}<0, \quad C^{2}-2 p X C-\left(1-X^{2}\right)>0 \text {. }
\end{aligned}
$$

Рассмотрим сначала случай “б”. Условие $1-6 X^{2}+X^{4}<0$ предполагает, что значения $X$ лежат в интервале

$$
X^{\prime}=\sqrt{3-2 \sqrt{2}} \approx 0.41<X<X^{\prime \prime}=\sqrt{3+2 \sqrt{2}} \approx 2.41 .
$$

Но начальное значение $X=0$ в этот интервал не попадает, и, следовательно, вариант “б” должен быть отвергнут.

Обратимся теперь к варианту “а". Условие $C^{2}-2 p X C-\left(1-X^{2}\right)<0$ может быть выполнено только при

$$
0<C<C_{\max }=p X+\sqrt{p^{2} X^{2}+\left(1-X^{2}\right)},
$$

где $C_{\mathrm{max}}$ - решение квадратного уравнения $C^{2}-2 p X C-\left(1-X^{2}\right)=0$. Предположим, что состояние с $C=C_{\text {max }}$ является регулярной точкой дисперсионных уравнений. В этом случае решение должно сушествовать и при $C>C_{\mathrm{max}}$, причем оно должно удовлетворять условию $K \geqslant 0$. Но для этого необходимо, чтобы одновременно с $C^{2}-2 p X C-\left(1-X^{2}\right)=0$ сомножитель $1-6 X^{2}+X^{4}$ также изменил свой знак. Последнее возможно только при условии, что при $C=C_{\max }$ коэффициент затухания $X=X_{\max }$ удовлетворяет уравнению $1-6 X^{2}+X^{4}=0$. Но, как легко убедиться с помошью дисперсионного уравнения (42), при $C^{2}-2 p X C-\left(1-X^{2}\right)=0$ и $1-6 X^{2}+X^{4}=0$ волновой вектор $K^{(2)}=0$. Так как это значение $K$ не совместимо со вторым дисперсионным 
уравнением (42), то состояние с $C=C_{\max }, X=X_{\max }$ не может быть решением системы дисперсионных уравнений. Поэтому, если при нулевой частоте решение удовлетворяло условиям $C=1, X=0$, то вьйти за границу $C_{\max }, X_{\max }$ оно уже никогда не сможет. Таким образом, всегда должны удовлетворяться неравенства

$$
0 \leqslant X<X_{\max }=\sqrt{3-2 \sqrt{2}} \approx 0.414, \quad 0<C<C_{\max }
$$

или в размерных переменных

$$
0 \leqslant x<x_{\max }=0.414 k, \quad 0<c<c_{\max }=c_{0}\left[0.414 p+\sqrt{0.16 p^{2}+0.86}\right] .
$$

Значение $c_{\max }$ в (46) мы получили, подставив в (46) $X=X_{\max }=0.414$.

Легко показать, что в интервале $X_{\max } \leqslant X \leqslant 1$ дисперсионные уравнения решений не имеют. В то же время при $X>1$ такое решение может появиться, что указывает на возможность существования второго звука. Но так как в этом случае $x>k$, то второй звук должен затухать на расстояниях порядка одной длины волны. Поэтому его весьма трудно наблюдать. Однако на генерирование второго звука источник должен тратить определенную энергию, что, возможно, позволит обнаружить предсказываемый эффект.

\section{6. ГИПЕРЗВУКОВЫЕ ЧАСТОТЫ}

Естественно предположить, что вблизи границы применимости модели сплошной среды или скорость звука $c$, или коэффициент затухания $x$ обрашаются в нуль (согласно приведенным выше неравенствам обратиться в бесконечность они не могут ни при каких условиях). Легко сообразить, что случай $c \rightarrow 0$ несовместим с дисперсионными уравнениями, так как из уравнения (41) ему соответствует одно значение коэффициента затухания $K$, а из (42) - другое. Поэтому рассмотрим случай $X \rightarrow 0$. В этом пределе дисперсионные уравнения (41), (42) вырождаются в уравнения

$$
\left(q-p C^{2}\right) K=2 C\left(1+2 K^{2}\right) X, \quad C^{3}-\left(1+K^{2}\right) C+4 q K=2 p C^{2} X,
$$

откуда получаем

$$
X^{(1)}=\frac{q-p C^{2}}{2 C\left(1+2 K^{2}\right)} K, \quad X^{(2)}=\frac{C^{3}-\left(1+K^{2}\right) C+4 q K}{2 p C^{2}} .
$$

Из первого уравнения (48) находим, что $X=0$ при

$$
C_{\infty}=\sqrt{\frac{q}{p}} \approx \sqrt{\frac{\eta}{a+\eta}}
$$

а из второго -

$$
K_{\infty}=\frac{1}{C_{\infty}}\left(2 q-\sqrt{4 q^{2}-C_{\infty}^{2}\left(1-C_{\infty}^{2}\right)}\right) .
$$

Отсюда следует, что сушествует некоторая предельная частота $\omega_{\infty}=k_{\infty} c_{\infty}$, выше которой коэффициент затухания становится отрицательным, что, конечно, несовместимо с законом сохранения энергии. По всей вероятности, именно значения $C_{\infty}, K_{\infty}$ и $X_{\infty}$ 
определяют границу применимости уравнений гидродинамики. Впрочем, не исключено, что исчезновение решения дисперсионных уравнений происходит при $\omega<\omega_{\infty}$ и связано не с обрашением в нуль коэффициента затухания, а скажем, с преврашением действительных решений дисперсионных уравнений в комплексные. Однако установить это можно только при численном решении дисперсионных уравнений. Во всяком случае, приведенные выше значения $C_{\infty}, K_{\infty}$ и $X_{\infty}$ определяют абсолютную границу применимости теории, перейти которую невозможно ни при каких условиях.

\section{7. ЗАКЛЮЧЕНИЕ}

Итак, сформулировав условия (1) применимости уравнений гидродинамики, мы обнаружили, что гидродинамическая теория звука должна быть справедливой вплоть до частот порядка $\omega \leqslant 10^{12} \mathrm{c}^{-1}$, т.е. должна охватывать практически весь интервал частот, представляюший интерес для эксперимента. Однако до сих пор этот вывод противоречил опытным данным. Подобное расхождение может быть объяснено только одним: уравнения гидродинамики, имеющие смысл законов сохранения массы, импульса и энергии, записаны не совсем точно. И действительно, проведенный анализ показал, что в уравнении теплопроводности отсутствует член, описывающий процесс переноса потенциальной энергии. Введение его в уравнение баланса внутренней энергии позволило устранить два приниипиальных расхождения между теорией и экспериментом: оказалось, что гидродинамическая теория правильно предсказывает зависимость коэффициента затухания от вязкости (затухание остается конечным во всем интервале изменения вязкости от $\eta=0$ до $\eta=\infty)$ и зависимость скорости звука от частоты (скорость звука остается конечной при всех частотах). Вопрос о том, позволяет ли теория правильно описывать конкретные зависимости коэффициентов затухания и скорости звука от различных параметров среды, требует специального исследования и сейчас решен быть не может. Однако уже сейчас теория может предсказать ряд новых эффектов, например исчезновение решений уравнений гидродинамики при некоторой максимальной частоте или возникновение второго звука с очень сильным затуханием. Но, пожалуй, наиболее интересным является утверждение, что безразмерное дисперсионное уравнение практически зависит только от одного материального параметра $q=\sqrt{\eta / a}$, где $\eta$-вязкость и $a-$ коэффициент температуропроводности. Это делает возможным построение универсальных зависимостей скорости звука $C=C(K, q)$ и коэффициента затухания $X=X(K, q)$ от волнового вектора $K$.

Естественно возникает вопрос, как соотносятся эти выводы с другими известными теоретическими результатами. Что касается релаксационной теории, то какое-либо соответствие установить трудно, поскольку каждая из двух теорий объясняет одни и те же факты разными причинами: в релаксационной теории предполагается, что традиционное уравнение теплопроводности является правильным, а все наблюдаемые несоответствия объясняются зависимостью теплоемкости от частоты, тогда как развитая выше гидродинамическая теория исходит из предположения, что в уравнении теплопроводности пропущен член, описывающий перенос потенциальной энергии. В релаксационной теории путем подгонки параметров (число которых, вообше говоря, не ограничено) можно добиться согласия с экспериментом. Можно надеяться, что такого же согласия удастся добиться и в гидродинамической теории. А если так, то судить о том, какая из 
них является более правильной, нужно не по эксперименту, а опираясь только на общетеоретические соображения. Сложнее обстоит дело с теорией, основанной на кинетическом уравнении Больцмана.

При сопоставлении кинетической и гидродинамической теорий звука необходимо иметь в виду, что:

а) уравнение Больцмана справедливо только в случае разреженных газов, тогда как уравнения гидродинамики описывают процессы в любой среде: в газах, жидкостях и в кристаллах (в последнем случае необходим учет анизотропии кристалла). Поэтому для того, чтобы сравнить предсказания обеих теорий, нужно сначала разложить гидродинамическое дисперсионное уравнение в ряд по степеням плотности, что само по себе представляет пока что нерешенную задачу;

б) уравнение Больцмана является приближенным, в то время как неравновесная иерархия ББГКИ, из которой в приближении локального равновесия были получены уравнения гидродинамики, является точным следствием уравнений движения классической механики. Поэтому согласие между двумя теориями не может быть абсолютным. Расхождение меж ду ними должно определяться той точностью, с которой уравнение Больцмана аппроксимирует иерархию ББГКИ в случае разреженных газов;

в) поток потенциальной энергии, который выше мы ввели в уравнение теплопроводности на основе эвристических соображений, следует из неравновесной иерархии ББГКИ в первом порядке по параметру $\varepsilon=R / L \approx 10^{-8}$, где $R$ - радиус корреляции, $L$ - характерный макроскопический размер [5]. В то же время аналогичный член может быть получен из уравнения Больцмана только в третьем приближении по параметру Кнудсена [7]. Как согласовать эти два различных способа разложений, не очень понятно. Кроме того, насколько нам известно, в кинетической теории задача о распространении звука с точностью до третьего порядка по параметру Кнудсена не решалась. Все это указывает на то, что сравнивать результаты, полученные в гидродинамической и кинетической теориях распространения звука, пока что рано.

\section{Список литературы}

[1] Л. Д. Ландау, Е. М. Лифииц. Механика сплошных сред. М.: Гостехиздат, 1954.

[2] C. Truesdell. J. Rat. Mech. Analys. 1953. V. 2. P. 643.

[3] Д. Сетте. Изучение простых жидкостей ультразвуковыми методами. В сб.: Физика простых жидкостей. Ред. Г. Темперли, Дж. Роулинсон, Дж. Рашбрук. М.: Мир, 1973. С. 151.

[4] Джс. Уленбек, Джс. Форд. Лекции по статистической механике. М.: Мир, 1965.

[5] Г. А. Мартынов. УФН. 1996. Т. 166. Вып. 10. С. 1105.

[6] Л. Д. Ландау, Е. М. Лифииц. Статистическая физика. Часть 1. М.: Наука, 1976.

[7] C. Чепмен, Т. Каулинг. Математическая теория неоднородных газов. М.: Иностранная литература, 1960.

[8] Ю. Л. Климонтович. Статистическая теория открытых систем. Т. 1. М.: Янус, 1995.

Поступила в редакцию 22.XII.2000 г., после доработки 12.II.2001 г. 